\title{
Measurements for Space in the A-Stub Muon System
}

\author{
ï. Cease \\ September 25, 1995
}

D-Zero Engineering Note: 3823.131-EN-432

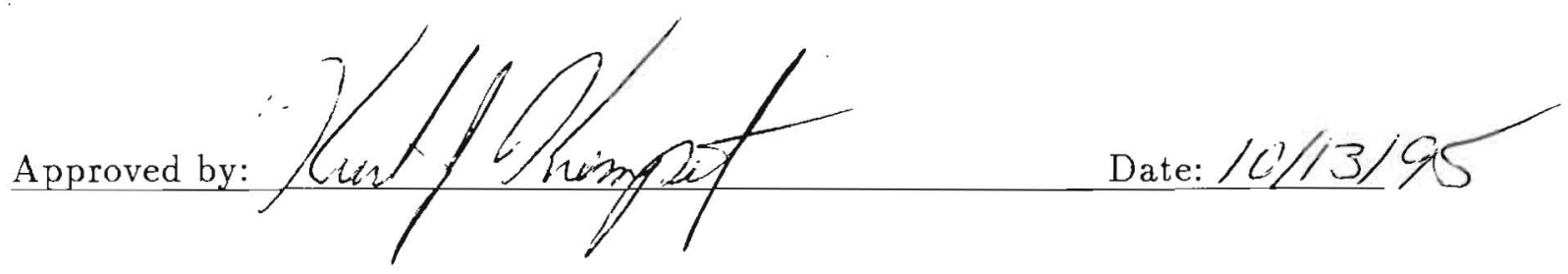




\title{
Measurements for Space in the A-Stub Muon System
}

\author{
H. Cease
}

September 25, 1995

\section{Introduction}

Measurements are given for the A layer Stub counters of the D-Zero Muon system. The purpose of the measurements is to determine the amount of space available for the A-stub muon counters. The counters will be positioned in between the central A layer PDTs and the cryostats. The given measurements are taken from the A layer PDTs towards the cryostat around the central portion of the detector. Drawings of the position and depth of the obstructions in a 5 inch clear zone are given.

\section{Method of Measurement}

Measurements were taken with the end cryostats closed. The end and central iron positions were open. The displacement due to the opening of the iron is subtracted from the measurements. The amount of the displacement is 47 inches on both the East and West sides. Measurements are taken from the exposed surface of the PDT, to the obstruction. All obstructions with a clearance of less than 5 inches are noted. The position of the obstruction is noted with respect to the PDT. Features on the PDTs such as rivets, electrical hutches, and tooling balls are not recorded. The tolerance between the PDT and the obstruction is $1 / 8$ inch. The position of the obstruction has a greater tolerance.

All measurements were checked by Andy Kuwazaki.

\section{Measurements}

Drawings showing the positions of obstructions for the East, West and bottom A layer PDTs are given. 


\subsection{Side A-layer Clearances}

The measurements of the position of the obstruction are taken from the North end PDT edge. The vertical position is recorded from the top surface of the bottom A layer PDT.

The spacing above the catwalk is recorded with the catwalk in the lowered position. With the catwalk in the raised (and irons closed) position a minimum of 1 inch should be subtracted from these measurements. A suggestion for creating more space in this region is to remove the catwalks before closing the central irons.

The space near the bottom supports of the pig trough could be expanded by changing the supporting I-beams. This suggestion would have to be thought about carefully due to the risk of damaging the cryostat cables. Additionally, the central magnets could be shimmed to create additional clearance over the entire East and West sides.

\subsection{Bottom A-layer Clearances}

The bottom region above the PDTs has a nearly uniform spacing. The spacing is created by the bottom of the pig trough. On the inner side next to the pig troughs is an approximate 6 inch region of cable trays and small piping that is possibly moveable. At the ends of the PDT, a 2 inch wide by $1 / 2$ inch deep cross member bar protrudes closer to the PDTs.

\subsection{Top A-layer Clearances}

The top A layer has a clearance of 3 inches as it is presently. The removal of the catwalk gratings above the cryostat would give an additional 1 inch of clearance below the PDTs. Below the catwalks is another inch of clearance with 1 or 2 small electrical box protruding into the space. Assuming the gratings and the small electrical boxes can be moved, a total of 5 inches can be obtained.

\section{Drawings}

See attached. Additional photographs are also given to help clarify obstruction location on drawings. 


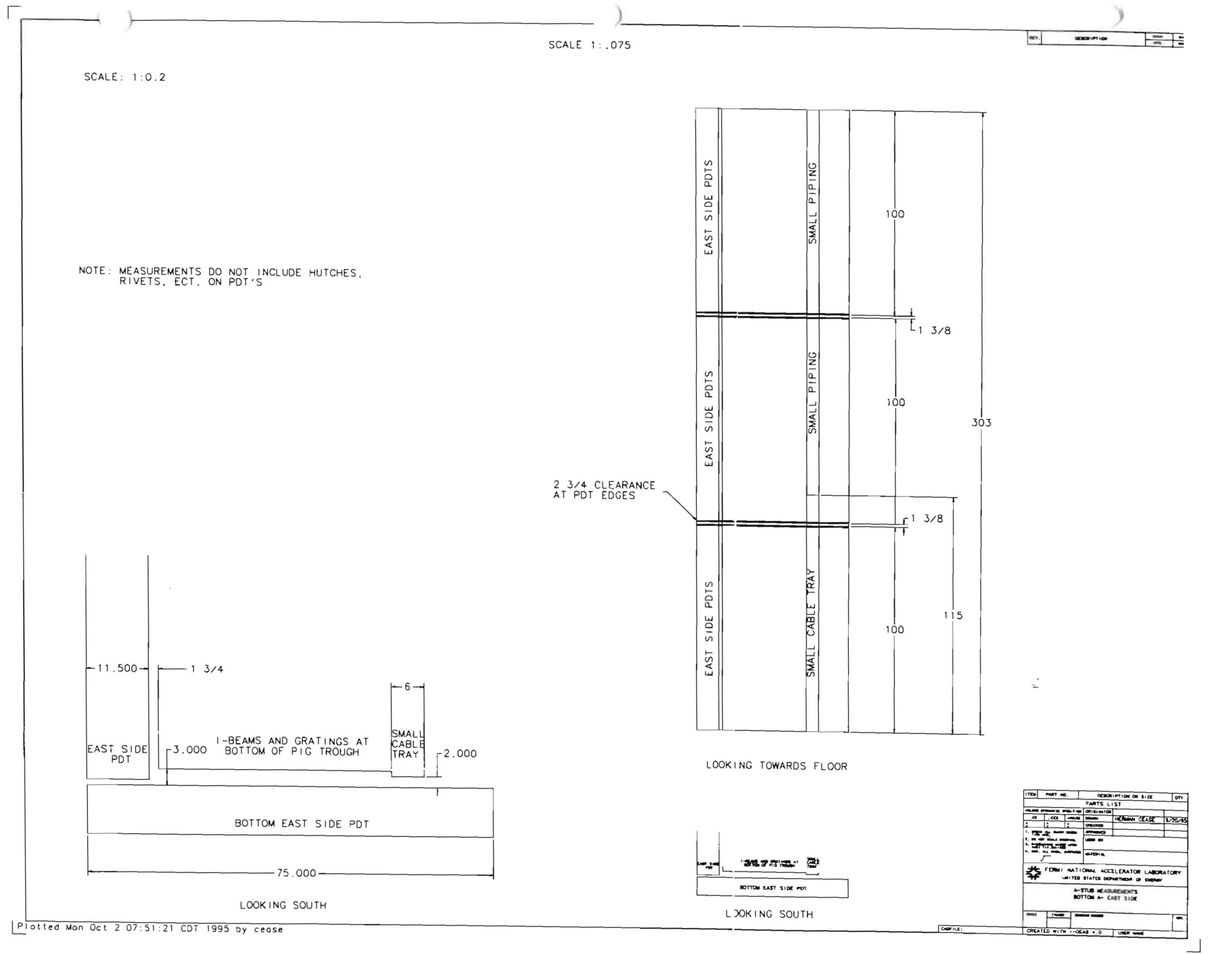


SCALE : $1: 0.2$

NOTE: MEASUREMENTS DO NOT INCLUDE HUTCHES,

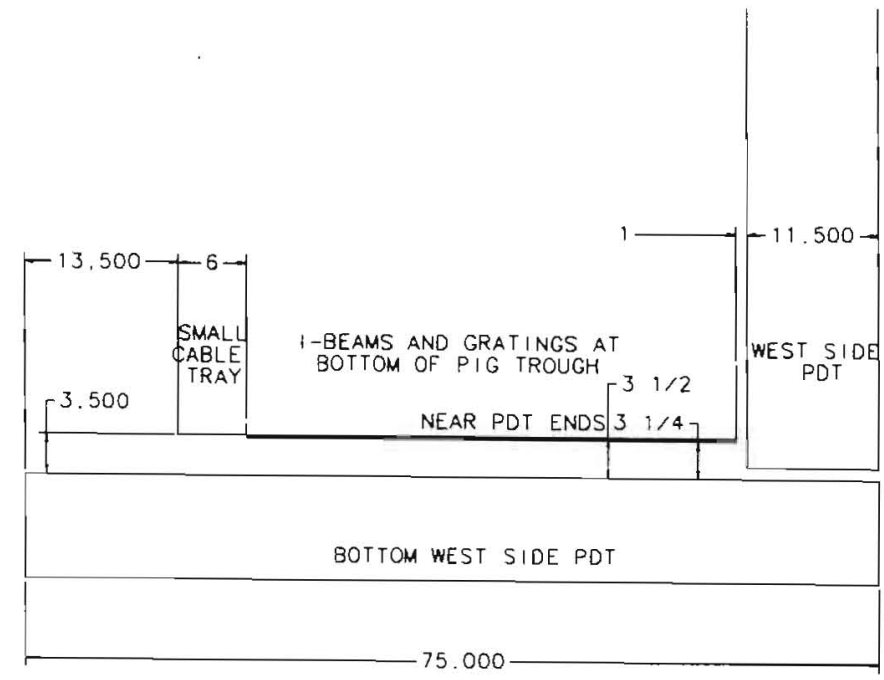

LOOKING SOUTH

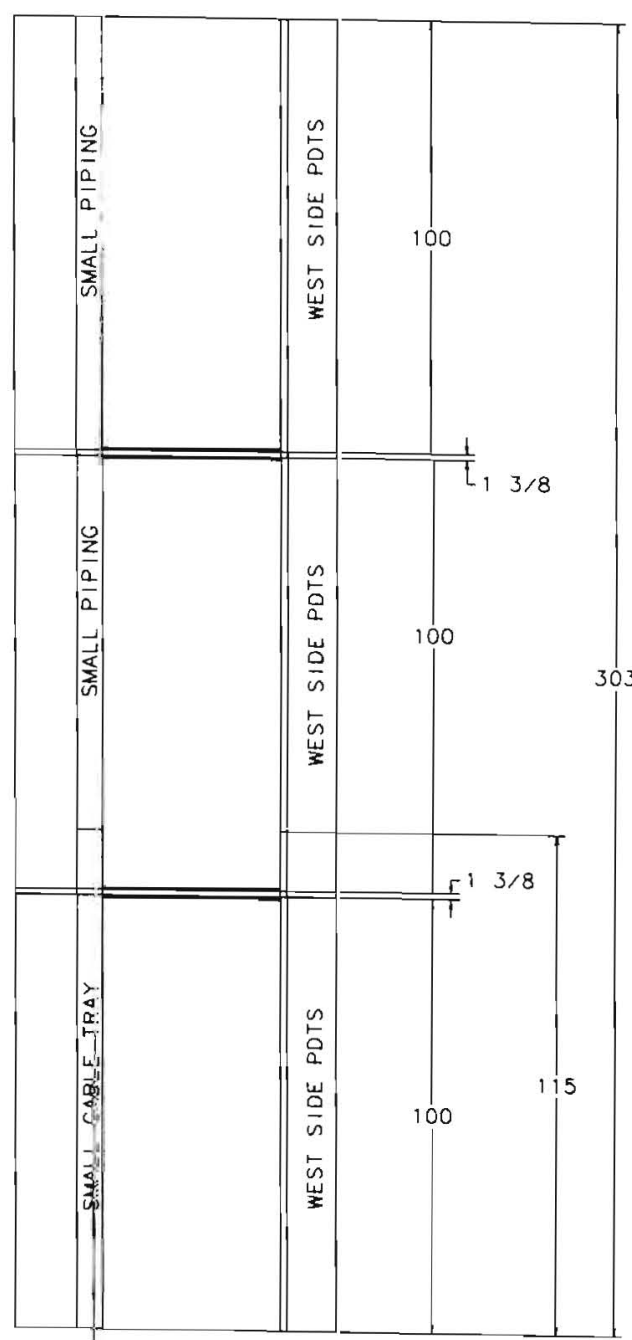

LOOK ING TOWARDS FLOOR

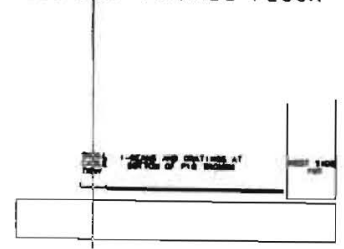

LOKING SOUTH

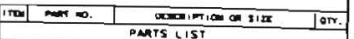

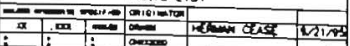
$-x-21$ $y=$

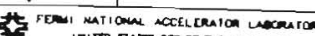
WEST SIDE BOTTOM A 


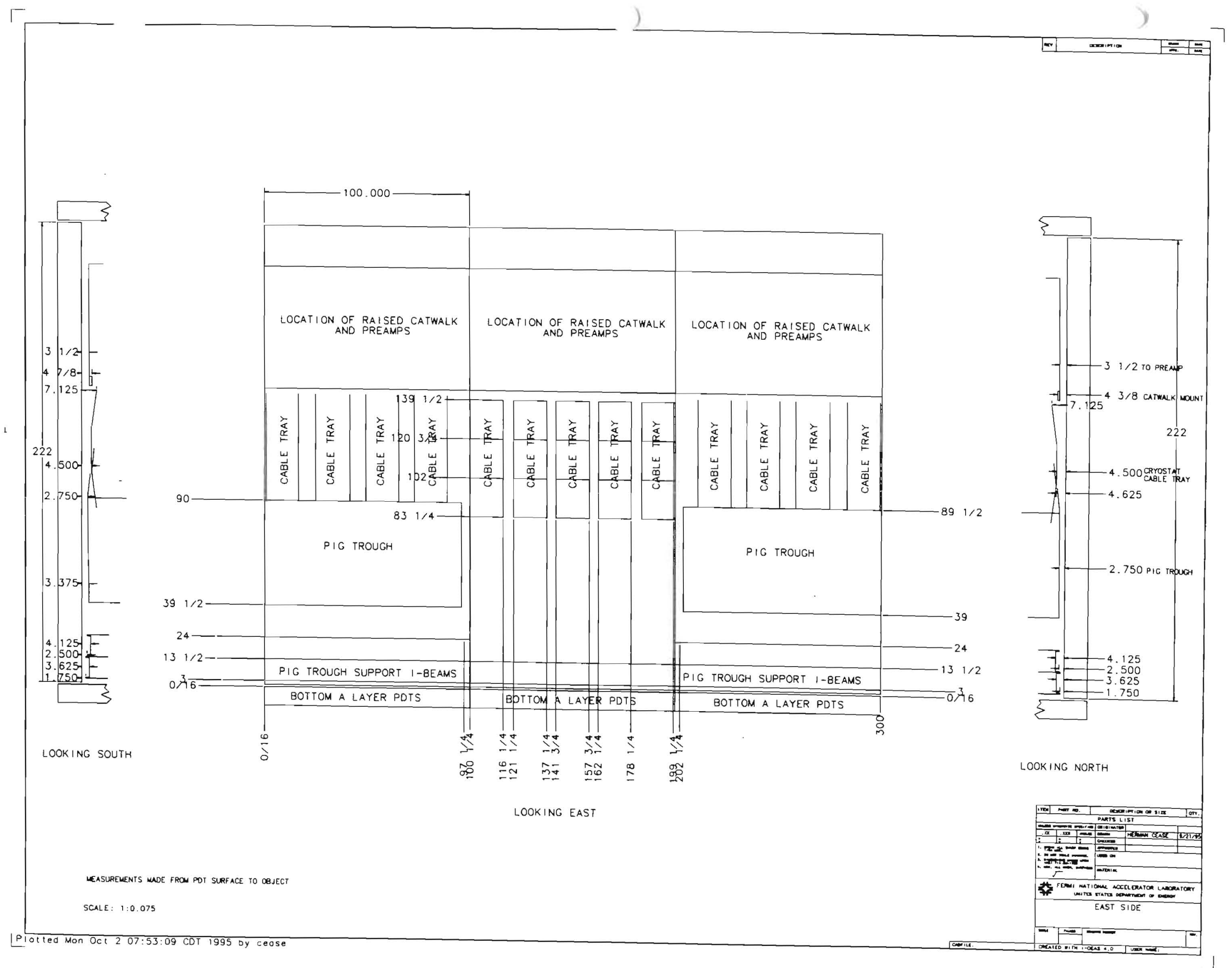




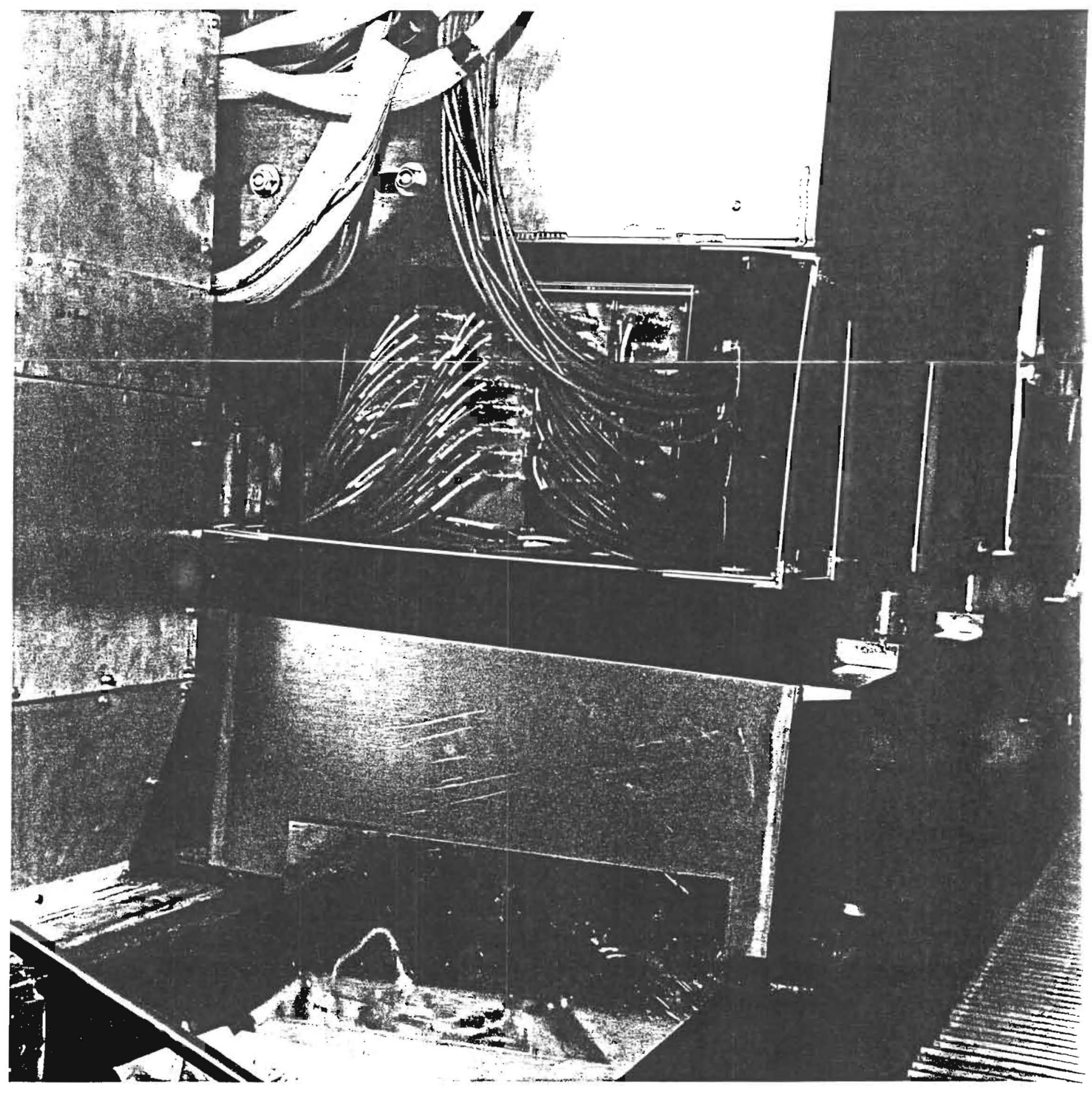

cable trays

arount er w-

Lower Pig Trough

Suprers 

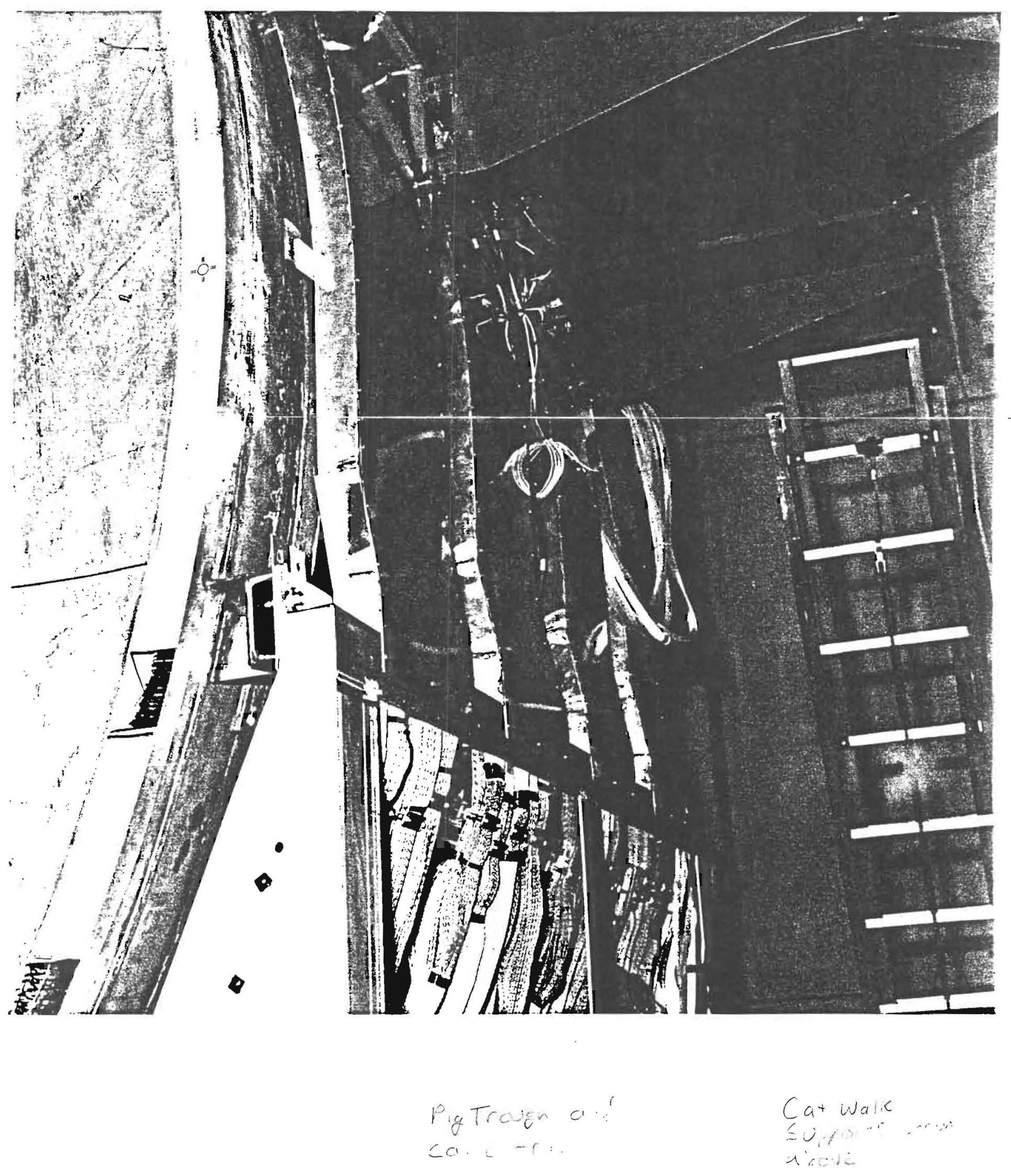
